Physicians should focus on risk stratification for patients with VTE, say the authors. Lifelong anticoagulation might be required for patients at high risk for recurrence, while the modest incremental reduction in risk seen after 6 months of anticoagulation might favor a shorter course of therapy for low-risk patients.

Caroline Barranco

Original article Ost D et al. (2005) Duration of anticoagulation following venous thromboembolism. JAMA 294: 706-715

\section{GM-CSF therapy for peripheral vascular disease}

Granulocyte-macrophage colony-stimulating factor (GM-CSF) is reported to stimulate arteriogenesis leading to improved collateral blood flow in patients with coronary artery disease. van Royen et al. have recently explored the use of this growth factor for treating patients with peripheral arterial disease.

Forty patients with moderate or severe intermittent claudication (vaso-occlusive disease of the legs) were randomly assigned to receive either placebo or subcutaneous treatment with GM-CSF for 14 days. Walking distances and ankle-brachial index were assessed on day 0 , 14 and 90. GM-CSF treatment was discontinued in three patients because of severe side effects (chest pain, muscle pain, hypotension and shortness of breath). Many other patients experienced less severe side effects such as skin rash and fever.

After 14 days, patients in both treatment arms could walk about $60 \mathrm{~m}$ further. The authors conclude that this was due to a large placebo effect, particularly as ankle-brachial index values did not increase significantly during the trial. White blood cell counts and levels of C-reactive protein increased significantly following GM-CSF therapy, and a temporary increase in the number of monocytes and CD34+ ${ }^{+}$stem cells was observed. Laser Doppler flowmetry measurements indicated that GM-CSF might be beneficial for the microcirculation, but the clinical relevance of this finding is unclear.

Noting that this study was limited by patient selection and, possibly, a suboptimal dosing scheme, the authors conclude that their data do not support the use of GM-CSF for treatment of patients with this condition.

Claire Braybrook

Original article van Royen $\mathrm{N}$ et al. (2005) A pilot study on stimulation of arteriogenesis using subcutaneous application of granulocyte-macrophage colony-stimulating factor as a new treatment for peripheral vascular disease. Circulation 112: $1040-1046$

\section{High cure rate for permanent atrial fibrillation with catheter ablation}

Catheter radiofrequency $(\mathrm{RF})$ ablation is known to be a feasible treatment for atrial fibrillation (AF), but most studies have been carried out in heterogeneous patient groups. A UK-based group has shown that maintenance of sinus rhythm can be achieved with catheter RF ablation in most patients with longstanding, permanent AF that does not respond to cardioversion or antiarrhythmic drugs, although half will need at least one repeat procedure.

This single-center study enrolled 42 consecutive patients (36 men). RF ablation was performed, with isolation of the pulmonaryvein ostia and three additional ablation lines in the left atrium. During follow-up (range 2-29 months), one patient died of an unrelated condition. Sinus rhythm was achieved in 31 of 41 patients, 22 of whom required at least one repeat procedure. Two patients suffered major complications; one had a nonfatal stroke attributed to char on the catheter (causing irrigated ablation catheters to be used for subsequent patients), and the second had a pulmonary-vein stenosis that required angioplasty. Half of the patients had an episode of atrial tachycardia, which might have been the result of incomplete ablation lines, a contributory factor in the high recurrence rate.

Catheter ablation is, therefore, an effective means of attaining sinus rhythm in patients with permanent $A F$, although it remains to be seen whether treatment confers a mortality benefit. There is a high recurrence rate, especially in patients with AF of long duration, but repeat procedures have a good chance of success.

Caroline Barranco

Original article Earley MJ et al. (2005) Catheter ablation of permanent atrial fibrillation: medium term results. Heart [doi 10.1136/hrt.2005.066969] 